\title{
A Phenotype of Atypical Apraxia of Speech in a Family Carrying SQSTM1 Mutation
}

\author{
Claire Boutoleau-Bretonnière ${ }^{\mathrm{a}, \mathrm{b}, \mathrm{c}, *}$, Agnès Camuzat $^{\mathrm{d}, \mathrm{e}, \mathrm{f}}$, Isabelle Le Ber ${ }^{\mathrm{d}, \mathrm{e}, \mathrm{f}, \mathrm{g}, \mathrm{h}}$, \\ Kawtar Bouya-Ahmed ${ }^{\text {d,e,f }}$, Rita Guerreiro ${ }^{\mathrm{i}}$, Anne-Laure Deruet ${ }^{\mathrm{a}, \mathrm{b}}$, Christelle Evrard ${ }^{\mathrm{a}, \mathrm{b}}$, José Bras ${ }^{\mathrm{i}}$, \\ Estelle Lamy ${ }^{\mathrm{a}, \mathrm{b}}$, Elisabeth Auffray-Calvier ${ }^{\mathrm{j}}$, Amandine Pallardy ${ }^{\mathrm{k}}$, John Hardy ${ }^{\mathrm{i}}$, Alexis Brice ${ }^{\mathrm{d}, \mathrm{e}, \mathrm{f}}$, \\ Pascal Derkinderen ${ }^{\mathrm{a}, \mathrm{b}, 1}$ and Martine Vercelletto ${ }^{\mathrm{a}, \mathrm{b}}$ \\ ${ }^{\mathrm{a}}$ CHU Nantes, Centre de Mémoire et de Ressource et Recherche (CM2R), Nantes, France \\ ${ }^{\mathrm{b}}$ Inserm, CIC 04, Nantes, France \\ ${ }^{\mathrm{c}}$ Laboratoire d'études des mécanismes cognitifs, EA 3082, Université Lyon 2, Bron F-69500, France \\ dinserm, UMR_S1127, CRICM, F-75013, Paris, France \\ ${ }^{\mathrm{e}}$ UPMC Univ Paris 06, UMR_S975, F-75013, Paris, France \\ ${ }^{\mathrm{f}}$ CNRS UMR 7225, F-75013, Paris, France

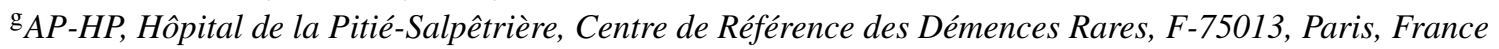

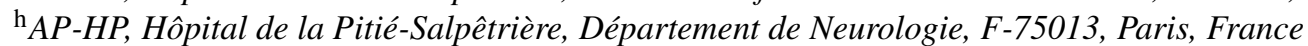 \\ ${ }^{i}$ Department of Molecular Neuroscience, UCL Institute of Neurology, London, UK \\ ${ }^{\mathrm{j}}$ Service de Neuroradiologie, CHU de Nantes, Nantes, France \\ ${ }^{\mathrm{k}}$ Service de Médecine Nucléaire, CHU de Nantes, Nantes, France \\ ${ }^{1}$ Inserm, UMR 913, Nantes, France
}

Accepted 23 June 2014

\begin{abstract}
SQSTM1 mutations, coding for the p62 protein, were identified as a monogenic cause of Paget disease of bone and of amyotrophic lateral sclerosis. More recently, SQSTM1 mutations were identified in few families with frontotemporal dementia. We report a new family carrying SQSTMI mutation and presenting with a clinical phenotype of speech apraxia or atypical behavioral disorders, associated with early visuo-contructional deficits. This study further supports the implication of SQSTM1 in frontotemporal dementia, and enlarges the phenotypic spectrum associated with SQSTM1 mutations.
\end{abstract}

Keywords: Amyotrophic lateral sclerosis, apraxia of speech, behavioral variant of FTD, frontotemporal lobar degeneration, non fluent variant of primary progressive aphasia, p62, Paget disease of bone, progressive non-fluent aphasia, progressive supranuclear palsy, SQSTM1

\section{INTRODUCTION}

Frontotemporal degeneration (FTD) defines a heterogeneous group of disorders that are interrelated. Clinical consensus criteria defined three variants according to the presenting symptoms: progressive

\footnotetext{
*Correspondence to: Claire Boutoleau-Bretonnière, Centre Mémoire Recherche et Ressources (CMRR), Clinique Neurologique CHU Nord, 44093 Nantes, France. Tel.: +33 240165 422; Fax: +33 240165 397; E-mail: claire.boutoleau-bretonniere@chu-nantes.fr.
}

behavioral changes in the behavioral variant of FTD (bvFTD), and prominent linguistic impairment in agrammatic (nonfluent/agrammatic primary progressive aphasia, PNFA) and semantic variants $[1,2]$. The definite diagnosis is based on three major pathological subtypes of frontotemporal lobar degeneration (FTLD) characterized by the presence of TDP43 (FTLDTDP), tau (FTLD-tau), or FUS-positive neuronal inclusions [3].

Thirty percent of FTD patients have a positive family history of the disease with an autosomal dominant 
inheritance. Mutations in the SQSTMI gene, coding for the p62 (sequestosome 1) protein, were initially identified as a cause of Paget's disease of bone (PDB) and, recently, as a cause of familial amyotrophic lateral sclerosis (ALS) [4]. More recently, SQSTM1 mutations were also identified in few families with autosomal dominant bvFTD [5, 6]. We described here a new clinical phenotype associated to SQSTM1 mutation.

\section{PATIENTS AND METHODS}

\section{Patients}

A French family has been studied (Fig. 1). Three siblings developed FTD at age ranging from 70 to 75 (patients 007, 008, 009). Their parents died at the age of 58 and 44 without known neurological disease. Family history was censored in both parental branches.

\section{Molecular analyses}

Blood samples of the three patients $(007,008,009)$ were obtained; all participants signed an informed consent for genetic studies. This study was approved by the local research Ethics Committee (Ethics Committee from Assistance-Publique Hôpitaux de Paris). DNA was extracted from blood samples. Mutations in known FTD (C9ORF72, MAPT, PGRN, $V C P, C H M P 2 B)$ and ALS genes (SOD1, TARDBP, FUS/TLS, PFN1, UBQLN2, hnRNPA2/B1, hnRNPA1, $M A T R 3)$ were initially excluded by Sanger sequencing or by repeat-primed PCR (for C9ORF72). The whole exome was sequenced on DNA samples of the three patients. Briefly, genomic DNA of patients was prepared according to Illumina's TruSeq Sample Preparation v3 (Illumina, CA, USA) and sequence capture, enrichment, and elution was performed according

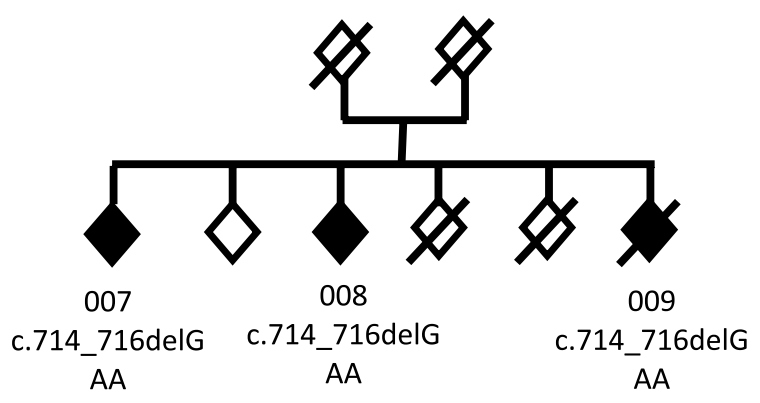

Fig. 1. Pedigree. The individuals are represented by diamonds for confidentiality. Black diamonds: FTD; white diamonds: non symptomatic individuals. Individuals 0007, 008, and 009 carried the c.714_716delGAA mutation. to manufacturer's protocols (Illumina's TruSeq Exome Enrichment). Sequencing was performed on Illumina's HiSeq2000 using 100 bp paired-end reads. Sequence alignment and variant calling was performed against the reference human genome (UCSC hg19) using bwa and the Genome Analysis Toolkit. Polymorphisms found in the public databases and in 50 in-house exomes of controls were excluded. Secondarily, mutations identified in SQSTM1 gene were validated by Sanger sequencing according to previously described methods [6]. The entire coding sequence was also sequenced in 350 French age-matched healthy controls.

\section{RESULTS}

\section{Molecular analyses}

A heterozygous mutation, c.714_716delGAA, p.Lys238del, was identified in the exon 5 of SQSTM1 gene in the three affected siblings (007, 008, 009). The mutation was validated by the Sanger method in the three patients (Fig. 1). The Lys238 residue is conserved across species. The mutation was previously identified as a disease-causing mutation in ALS patients [4] and was predicted to be deleterious by SIFT/PROVEAN in silico software (score: -11.7 ). Furthermore, this mutation was absent from 350 French controls, from 6,503 individuals of the 'exome variant server' (http://evs.gs.washington.edu/EVS/), dbSNP (http://www.ncbi.nlm.nih.gov/projects/SNP/) and 1000Genomes (http://www.1000genomes.org/) databases, thus strongly supporting its deleterious effect.

\section{Clinical features}

Patients' neuropsychological scores are summarized in Table 1.

\section{Proband 007}

This 77-year-old right-handed patient was evaluated in our Department after two years of language difficulty. He had endoprothetic treatment of the left hip at 67 years old and a right femoral fracture at the age of 75, without any obvious radiological or biological signs of PDB. Examination showed apraxia of speech characterized by an articulation planning deficit, dysarthria with hypokinetic features and buccofacial apraxia, associated with semantic and phonetic paraphasia. Phonemic and semantic fluency sores were low 
Table 1

Neuropsychological scores of the patients

\begin{tabular}{|c|c|c|c|c|c|c|}
\hline & Patient 007 & & Patient 008 & & Patient 009 & \\
\hline Age at evaluation, $\mathrm{y}$ & 77 & 82 & 71 & 79 & 74 & 76 \\
\hline Disease duration, $\mathrm{y}$ & 2 & 7 & 1 & 9 & 2 & 4 \\
\hline $\mathrm{MMS} / 30$ & 23 & 14 & 24 & 24 & 18 & 11 \\
\hline \multicolumn{7}{|l|}{ FCSRT } \\
\hline Encoding/16 & $12 *$ & $13^{*}$ & 16 & $13^{*}$ & $14^{*}$ & NA \\
\hline Free recall $1,2,3 / 16$ & $5 / 6 / 7$ & $4 / 3 / 4 *$ & $6 / 9 / 9$ & $6 / 7 / 8$ & $5 / 5 / 9$ & NA \\
\hline Cued recall $1,2,3 / 16$ & $11 / 10 / 9$ & $6 / 12 / 8$ & $10 / 7 / 7$ & $6 / 7 / 8$ & $10 / 10 / 7$ & NA \\
\hline Total recall/48 & 48 & $37 *$ & 48 & 42 & 46 & NA \\
\hline Recognition/16 & 16 & 16 & 16 & 16 & 16 & NA \\
\hline Delayed total recall & 15 & NA & NA & 16 & 15 & NA \\
\hline Rey figure Copy/36 & NA & NA & 19 & NA & 17 & 18 \\
\hline Oral confrontation naming/80 & $69^{*}$ & $61 *$ & 80 & 73 & 75 & 73 \\
\hline \multicolumn{7}{|l|}{ Verbal fluency 2 ' } \\
\hline P letter & $12 *$ & NA & 23 & 20 & $10 *$ & $9 *$ \\
\hline Animals & $2 *$ & NA & 20 & 30 & $6^{*}$ & $4 *$ \\
\hline Frontal Assessment Battery/18 & $5^{*}$ & $5^{*}$ & $15^{*}$ & 16 & $9 *$ & $3 *$ \\
\hline \multicolumn{7}{|l|}{ Wisconsin Card Sorting Test } \\
\hline Criteria/6 & $1 *$ & NA & $2 *$ & $2 *$ & NA & NA \\
\hline Errors & $21 *$ & NA & $20 *$ & $13^{*}$ & NA & NA \\
\hline Perseverations & $3^{*}$ & NA & $9 *$ & $5 *$ & NA & NA \\
\hline TMT A & NA & NA & $\mathrm{C} 0.75$ & C50 - 0.75 & $\mathrm{C} 0.75$ & $\mathrm{C} 0.75$ \\
\hline TMT B & NA & NA & Impossible* & Impossible* & Impossible* & Impossible* \\
\hline
\end{tabular}

MMS, Mini Mental Score; FCSRT, Free and Cued Selective Recall Reminding Test; TMT, Trail Making Test; NA: not available,*abnormal value.

(respectively 12 and 2 words in two minutes). Syntactic and single-word comprehensions were spared. Mild grammatical deficit appeared in his spoken picture description and in writing. Neuropsychological testing showed executive dysfunction associated with severe visuo-constructional impairment when copying the Rey figure but preservation of memory (Fig. 2, Table 1). Careful interview of the patient's caregiver revealed the absence of behavioral disorders. Deep tendon reflexes were increase in all limbs and plantar reflex was flexor bilaterally. He had no motor symptoms, no gaze limitation, and no parkinsonian signs. Brain magnetic resonance imaging (MRI) showed major diffuse cortical atrophy more marked on the frontal and temporal lobes. ECD single photon emission computed tomography (SPECT) of the brain revealed severe hypoperfusion of frontal and temporal lobes, predominantly on the right side, and hypoperfusion of basal ganglia (Fig. 3). Five years later, the patient displayed severe phonetic disintegration with worsening of agrammatism but comprehension was spared. There were no clinical or electromyographic signs of ALS.

\section{Sibling 008}

This 71-year-old right-handed patient developed apragmatism over several months, without others behavioral troubles. On initial examination, he had a moderate dysarthria due to buccofacial apraxia.
There were no signs of upper or lower motor neurons dysfunctions. Neuropsychological testing showed executive dysfunction associated with visuoconstructional deficit, without aphasia. Brain MRI showed major diffuse atrophy more prominent on the frontal and parietal lobes. Brain SPECT revealed severe hypoperfusion of frontal and parietal lobes, particularly in bilateral sulcus centralis (Fig. 3). Eight years after the disease onset, the clinical features were highly consistent with apraxia of speech associated with visuo-constructional deficit. Executive functions also deteriorated (Table 1), but autonomy was still preserved with an overall slow progression.

\section{Sibling 009}

This 74-year-old right-handed patient presented with visual and auditory hallucinations by the age of 72. He had bilateral hip fractures at the age of 67 caused by falls, but had no obvious clinical, biological, or radiological signs of PDB. Neurological examination was normal. Neuropsychological testing showed preserved memory functions with severe executive syndrome and visuo-constructional disabilities (Fig. 2). CT scan showed bilateral frontal atrophy. Brain ECD-SPECT revealed cortical frontal and temporal hypoperfusion (Fig. 3). Severe behavioral disorders subsequently appeared, especially self-mutilation that required hand amputation. Cognitive deficit worsened over time lead- 


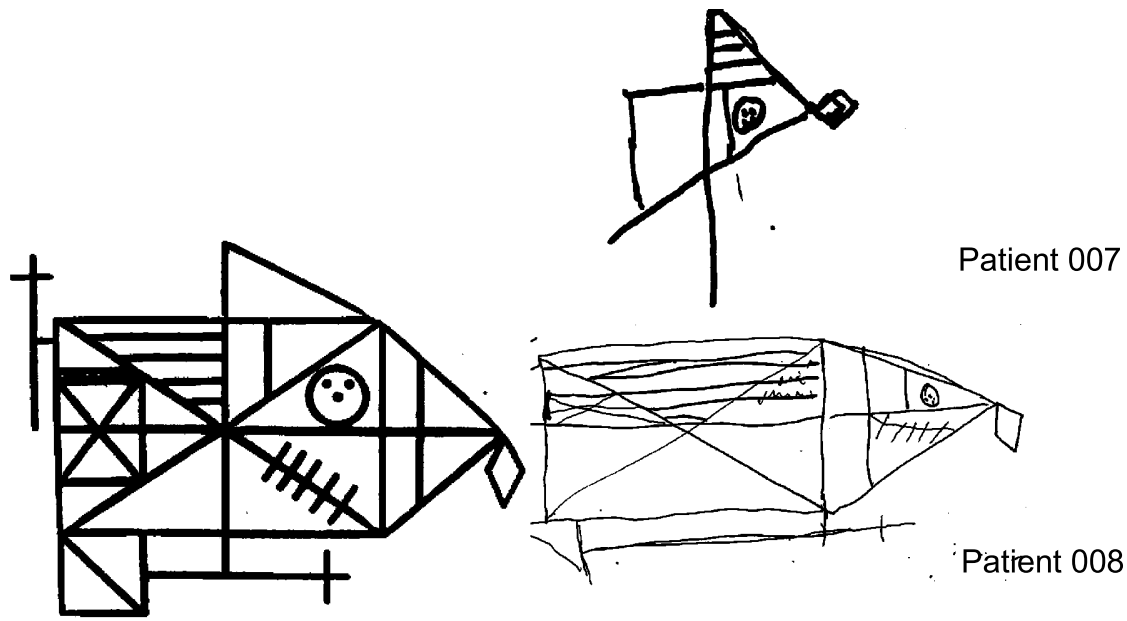

Rey Figure

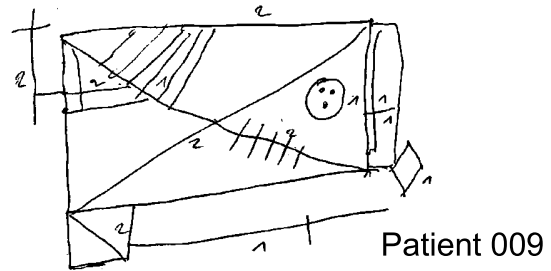

Fig. 2. Copy of Rey figure of the patients carrying SQSTM1 mutations

Patient 007
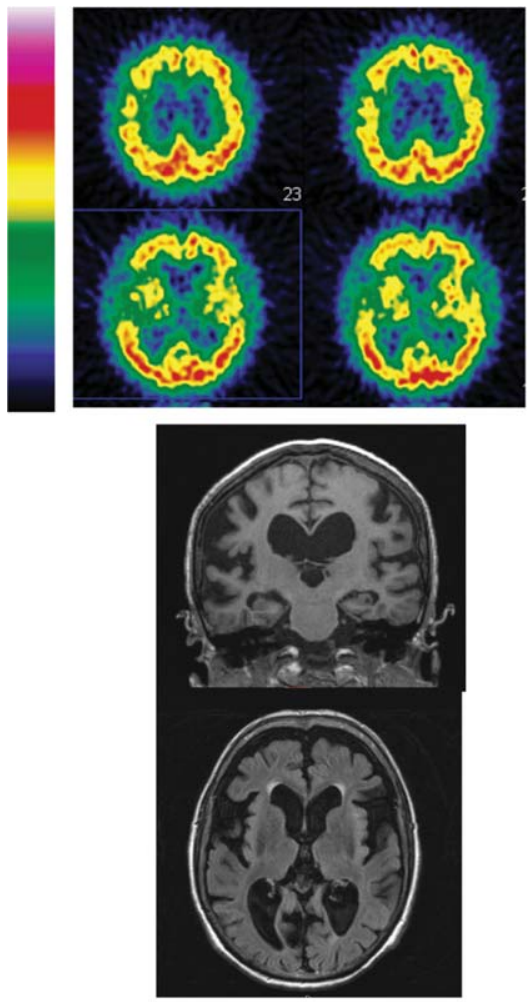

Patient 008
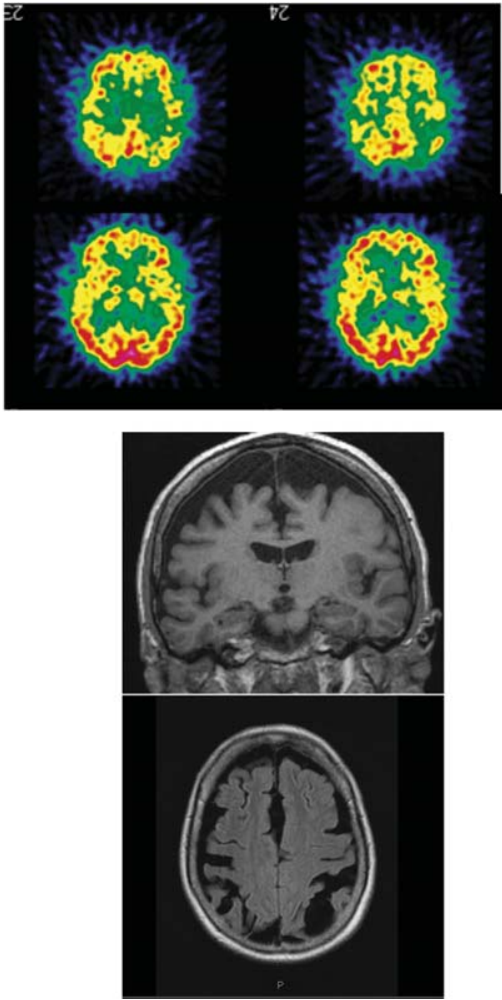

Patient 009

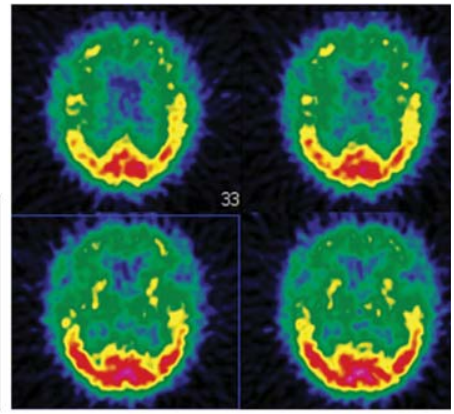

Fig. 3. Brain imaging. ECD-SPECT (top) and brain MRI (coronal and axial sections, bottom) of patients 007,008 , and 009. 
ing to the diagnosis of bvFTD. The patient was finally institutionalized and died at 80 years.

\section{DISCUSSION}

We describe a new family carrying a heterozygous p.Lys238del mutation in SQSTM1 gene segregating with FTD. This study further demonstrates the implication of SQSTM1 in FTLD spectrum of diseases. Clinically, the three patients of the family were affected by atypical forms of FTD. Two patients had hip and femoral fractures, but none presented severe bone symptoms and deformations. The patients were not investigated specifically with biological testing or bone scintigraphy but no radiological signs of PDB were present on radiography. Furthermore, although the p.Lys238del mutation has been previously identified in ALS patients [4], motor neuron symptoms were absent in the three patients. ALS patients are often not tested for FTD symptoms, which could be the case for the ALS patient with the same mutation. This family thus illustrates the variability of bone and motor phenotype that can be moderate or absent in SQSTM1 families. We propose that $S Q S T M 1$ gene should be integrated in the 'multisystem proteinopathies' (MSP4), an emerging group of genetic diseases clinically characterized by variable association of FTD, ALS, PDB, and myopathy, and so far including VCP (MSP1), hnRNPA2/B1 (MSP2), and hnNPA2 genes (MSP3) [7].

The three patients exhibited a similar neuropsychological profile associating dysexecutive syndrome and early visuo-constructional disabilities. The episodic memory was for a long time preserved. The patients were mainly apathetic and one initially presented psychotic symptoms with hallucinations at onset. Brain imaging showed major opercular and perisylvian involvement. None of the three patients completely fit with the clinical criteria of bvFTD of Rascovsky [2] at onset. Patient 009 was the only one to fulfil these criteria after several years of evolution.

More interestingly, patient 007 presented apraxia of speech, with buccofacial apraxia dysarthria and anomia at onset. The patient 008 also had moderate symptoms of apraxia of speech. Apraxia of speech can be the initial sign of PNFA [2]. However, even if two patients presented with language problems, both of them presented early visuo-constructional disorders and, therefore, do not completely fit with the diagnosis of primary progressive aphasia according to the criteria of Gorno-Tempini et al. [2,8]. They also showed different neuroimaging patterns, and neither of them presents the typical neuroimaging of nfv-PPA $[2,8]$.
Many genetic cases of FTD do not present with the typical features of sporadic FTD, or do not strictly meet the criteria for any of the clinical variants. Notably, in previous studies, all FTD patients carrying SQSTM1 mutations presented a behavioral variant of FTD [5, 6]. This family demonstrates that the phenotypes associated with SQTMI mutations are more heterogeneous, and that this gene should also be studied in patients presenting with speech apraxia or behavioral disorders associated with visuo-constructive deficit at onset, even in absence of PDB or ALS.

Predicting the underlying pathology in patients with FTD is difficult but will be particularly important for future treatments targeting tau or TDP-43 proteins. A pathological study previously showed that SQSTM1 mutation carriers had TDP-43 and p62immunoreactive neuronal inclusions in the prefrontal cortex [9]. So far, PNFA and more precisely, apraxia of speech, was rather considered predictive of FTLD-tau [10-12]. Our study supports a possible link between speech apraxia and FTLD-TDP. This finding is supported by a recent pathological study in 11 patients showing that nonfluent/agrammatic primary progressive aphasia could be associated with FTLD-TDP pathology [13].

In conclusion, our report widens the phenotypic spectrum of SQSTM1 mutations, and further illustrates the variability of phenotypes associated with SQSTM1 mutations thus supporting a pleiotropic effect of the mutations.

\section{ACKNOWLEDGMENTS}

This work was funded by the Neuromics FP7 contract E12009DD (to A.B.), the France Alzheimer Association contract R12091DD (to A.B.), 'The Programme Hospitalier de Recherche Clinique' (PHRC) (to I.L.B.), and from the program "Investissements d'avenir" ANR-10-IAIHU-06. This work was also funded in part, by Alzheimer's Research UK, by an anonymous donor, by the Wellcome Trust/MRC Joint Call in Neurodegeneration award (WT089698) to the UK Parkinson's Disease Consortium whose members are from the University College London/Institute of Neurology, the University of Sheffield, and the MRC Protein phosphorylation Unit at the University of Dundee, Scotland, and by a fellowship from Alzheimer's Research UK to Dr Guerreiro. We thank Ms. Lydia Guennec, Isabelle Lagroua, Sylvie Forlani and Christelle Dussert (DNA and cell bank of CRICM, Hôpital de la Salpêtrière, Paris) for their excellent technical assistance. 
Authors' disclosures available online (http://www.jalz.com/disclosures/view.php?id=2406).

\section{REFERENCES}

[1] Rascovsky K, Hodges JR, Knopman D, Mendez MF, Kramer JH, Neuhaus J, van Swieten JC, Seelaar H, Dopper EG, Onyike CU, Hillis AE, Josephs KA, Boeve BF, Kertesz A, Seeley WW, Rankin KP, Johnson JK, Gorno-Tempini ML, Rosen H, Prioleau-Latham CE, Lee A, Kipps CM, Lillo P, Piguet O, Rohrer JD, Rossor MN, Warren JD, Fox NC, Galasko D, Salmon DP, Black SE, Mesulam M, Weintraub S, Dickerson BC, Diehl-Schmid J, Pasquier F, Deramecourt V, Lebert F, Pijnenburg Y, Chow TW, Manes F, Grafman J, Cappa SF, Freedman M, Grossman M, Miller BL (2011) Sensitivity of revised diagnostic criteria for the behavioural variant of frontotemporal dementia. Brain 134, 2456-2477.

[2] Gorno-Tempini ML, Hillis AE, Weintraub S, Kertesz A, Mendez M, Cappa SF, Ogar JM, Rohrer JD, Black S, Boeve BF, Manes F, Dronkers NF, Vandenberghe R, Rascovsky K, Patterson K, Miller BL, Knopman DS, Hodges JR, Mesulam MM, Grossman M (2011) Classification of primary progressive aphasia and its variants. Neurology 76, 1006-1014.

[3] Mackenzie IR, Rademakers R, Neumann M (2010) TDP-43 and FUS in amyotrophic lateral sclerosis and frontotemporal dementia. Lancet Neurol 9, 995-1007.

[4] Fecto F, Yan J, Vemula SP, Liu E, Yang Y, Chen W, Zheng JG, Shi Y, Siddique N, Arrat H, Donkervoort S, Ajroud-Driss S, Sufit RL, Heller SL, Deng HX, Siddique T (2011) SQSTM1 mutations in familial and sporadic amyotrophic lateral sclerosis. Arch Neurol 68, 1440-1446.

[5] Rubino E, Rainero I, Chio A, Rogaeva E, Galimberti D, Fenoglio P, Grinberg Y, Isaia G, Calvo A, Gentile S, Bruni AC, St George-Hyslop PH, Scarpini E, Gallone S, Pinessi L (2012) SQSTM1 mutations in frontotemporal lobar degeneration and amyotrophic lateral sclerosis. Neurology 79, 1556-1562.

[6] Le Ber I, Camuzat A, Guerreiro R, Bouya-Ahmed K, Bras J, Nicolas G, Gabelle A, Didic M, De Septenville A, Millecamps S, Lenglet T, Latouche M, Kabashi E, Campion D, Hannequin D, Hardy J, Brice A (2013) SQSTM1 mutations in French patients with frontotemporal dementia or frontotemporal dementia with amyotrophic lateral sclerosis. JAMA Neurol 70, 1403-1410.

[7] Kim HJ, Kim NC, Wang YD, Scarborough EA, Moore J, Diaz Z, MacLea KS, Freibaum B, Li S, Molliex A, Kanagaraj AP, Carter R, Boylan KB, Wojtas AM, Rademakers R, Pinkus JL, Greenberg SA, Trojanowski JQ, Traynor BJ, Smith BN, Topp S, Gkazi AS, Miller J, Shaw CE, Kottlors M, Kirschner J, Pestronk A, Li YR, Ford AF, Gitler AD, Benatar M, King OD, Kimonis VE, Ross ED, Weihl CC, Shorter J, Taylor JP (2013) Mutations in prion-like domains in hnRNPA2B1 and hnRNPA1 cause multisystem proteinopathy and ALS. Nature 495, 467-473.

[8] Gorno-Tempini ML, Dronkers NF, Rankin KP, Ogar JM, Phengrasamy L, Rosen HJ, Johnson JK, Weiner MW, Miller BL (2004) Cognition and anatomy in three variants of primary progressive aphasia. Ann Neurol 55, 335-346.

[9] Teyssou E, Takeda T, Lebon V, Boillee S, Doukoure B, Bataillon G, Sazdovitch V, Cazeneuve C, Meininger V, LeGuern E, Salachas F, Seilhean D, Millecamps S (2013) Mutations in SQSTM1 encoding p62 in amyotrophic lateral sclerosis: Genetics and neuropathology. Acta Neuropathol 125, 511522.

[10] Josephs KA, Duffy JR, Strand EA, Whitwell JL, Layton KF, Parisi JE, Hauser MF, Witte RJ, Boeve BF, Knopman DS, Dickson DW, Jack CR, Jr, Petersen RC (2006) Clinicopathological and imaging correlates of progressive aphasia and apraxia of speech. Brain 129, 1385-1398.

[11] Grossman M (2010) Primary progressive aphasia: Clinicopathological correlations. Nat Rev Neurol 6, 88-97.

[12] Deramecourt V, Lebert F, Debachy B, Mackowiak-Cordoliani MA, Bombois S, Kerdraon O, Buee L, Maurage CA, Pasquier F (2010) Prediction of pathology in primary progressive language and speech disorders. Neurology 74, 42-49.

[13] Caso F, Mandelli ML, Henry M, Gesierich B, Bettcher BM, Ogar J, Filippi M, Comi G, Magnani G, Sidhu M, Trojanowski JQ, Huang EJ, Grinberg LT, Miller BL, Dronkers N, Seeley WW, Gorno-Tempini ML (2014) In vivo signatures of nonfluent/agrammatic primary progressive aphasia caused by FTLD pathology. Neurology 82, 239-247. 